\title{
Die (guten) Veränderungen in Medizin und Politik
}

\section{Jean Martin}

Dr. med., Mitglied der Redaktion

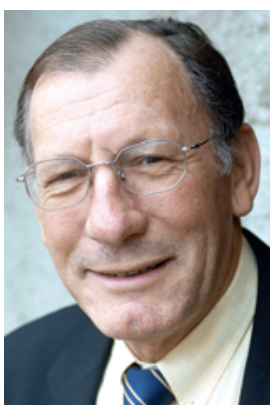

Literatur

1 Artikel von M. Eltchaninoff. Journal Le Point, Paris, 27. April 2017, S. $46-48$.

2 «Le simple patient a sa place dans la réflexion éthique». Médecins (Bulletin der Nationalen Ärztekammer, Paris), März-April 2017, Nr. 48 S. 32.

3 «Patients-experts, vers un nouveau métier?». Médecins, Nr. 48, S. 8-9

\footnotetext{
1 Ordre national français des médecins.

2 Comité consultatif national d'éthique.

3 Der Begriff «Experten-

patient» wird immer mehr auch in den Schweizer Medien verwendet. Diese Personen werden auch in der Praxis eingesetzt (so z.B. im Kanton Waadt in der Betreuung von Diabe tikern-siehe www.diabe tevaud.ch/patient-expert).
}

jean.martin[at]saez.ch
Der neu gewählte französische Präsident wurde von den Ideen des renommierten Philosophen Paul Ricœur inspiriert, dessen Assistent er einmal war. In einem Artikel, der just zum Zeitpunkt der Wahl erschien [1], stellt Michel Eltchaninoff fest, dass für Macron, der weder mit dem ultraliberalen Modell noch mit einem vereinheitlichenden Kollektivismus etwas am Hut hat, die Herausforderung nicht darin besteht, allen dasselbe zu geben, sondern jedem das zu bieten, was er braucht. Er ist der Ansicht, dass «Politik darin besteht, es den Menschen zu ermöglichen, ihr Potenzial zu entwickeln", und bevorzugt als Leitlinie Gerechtigkeit vor Gleichheit um jeden Preis.

«Ich glaube nicht», sagt er, "dass die Politik ein Glücksversprechen machen soll, denn sie kann nicht alles regeln oder alles verbessern. Sie sollte es vielmehr jedem ermöglichen, seinen Weg zu finden, sein Schicksal selbst in die Hand zu nehmen.» Angesichts dessen und im Hinblick auf die Ziele der Medizin und der Pflege stelle ich auffallende Analogien fest. Ersetzen Sie einmal «Politik» mit «Medizin», und es passt sehr gut. Ein Arzt kann nicht alles regeln, er ist da, um es seinen $\mathrm{Pa}$ tienten zu ermöglichen, ihr Potenzial (wo noch vorhanden) bestmöglich zu entwickeln, um noch selbst eine Wahl zu treffen (auch wenn die Auswahl nur noch begrenzt ist). Und es geht darum herauszufinden, was für jeden Patienten in seiner spezifischen Situation am besten ist.

Das jüngste Bulletin der französischen Ärztekammer ${ }^{1}$ enthält einen Text von Prof. Jean-François Delfraissy [2], dem neuen Vorsitzenden der Nationalen Ethikkommission $(\mathrm{CCNE})^{2}$. Er erlebte die dramatischen Ereignisse rund um AIDS seit dessen Ausbruch zu Beginn der Achtzigerjahre mit: «Wir waren eine Generation junger Ärzte, die sich mit etwas radikal Neuem konfrontiert sahen: Es gab keine zwei Seiten mehr, nämlich die des wissenden Mediziners und jene des unwissenden Kranken. Es gab Patienten, die aktiv und mit der Unterstützung (nichtmedizinischer) Verbände ihre Gesundheit managten. Etwas noch nie Dagewesenes.» Als Kantonsarzt erlebte ich diese Zeit intensiv mit. Dieses Phänomen des Sicherheitsverlustes einer vorerst machtlosen Medizin brachte eine Entwicklung in Gang, die mit dem vergleichbar ist, was der französische Präsident jetzt unter dem Einfluss von Ricœur, der sich intensiv mit Sozialethik auseinan- dergesetzt hat, in die Politik einbringen will. Nochmals Delfraissy: «Die fachliche Dimension unseres Berufs ist unabdingbar, bleibt aber unvollständig, wenn wir nicht fähig sind, den Menschen und - im grösseren Rahmen der Zivilgesellschaft zuzuhören. Dies möchte ich der CCNE vorschlagen, deren Mitglieder sicher rennommierte Experten sind. Der gesunde Menschenverstand jedoch, und somit der einfache Bürger, muss seinen Platz in unseren ethischen Überlegungen haben.»

Macron will den Bürger nicht als verwaltetes Wesen, sondern als vollwertigen Akteur sehen [1]. In der medizinischen und pflegerischen Praxis gibt es heutzutage eine solche Bewegung, die die Partnerschaft zwischen Patient und Arzt oder Pfleger sowie die gemeinsame Entscheidung betont.

Eine weitere Entwicklung, die im oben erwähnten Bulletin beschrieben wird: In Frankreich haben sogenannte «Universités des patients» [3] begonnen, Patienten zu Experten auszubilden - chronisch Kranke, die ihre Erfahrung anderen Kranken zugute kommen lassen (z.B. bei Diabetes, Multipler Sklerose, Niereninsuffizienz, Krebs usw.). Die Hochschule von Paris war 2010 die erste dieser Art, gefolgt von Marseille und Grenoble. Die Ausbildung eröffnet den Expertenpatienten ${ }^{3}$ die Möglichkeit, sich sozial und beruflich wieder zu integrieren. In Paris haben schon 130 Personen ihr Abschlussdiplom erhalten. Wenn auch eine gewisse Anzahl eine Anstellung, hauptsächlich in der therapeutischen Ausbildung, gefunden haben, «liegt noch ein langer Weg vor ihnen. Im Spital hat der gelehrte Arzt weiterhin die Tendenz, sich dem nichtfachlichen Patienten zu widersetzen». Ich weiss von keiner solchen formalisierten Initiative in der Schweiz, aber der Artikel erwähnt: «Das Konzept ist nicht neu. In Kanada, den USA oder der Schweiz werden Patienten schon seit langem beigezogen.» Es stimmt, dass sich bei uns verschiedene Patientenverbände schon seit langem mit eigenen Beiträgen in die Betreuung - und die Interessenverteidigung - chronisch Kranker einbringen.

Zum Schluss ein Ratschlag Macrons: «Wir haben uns gleichsam in traurigen Eifer, Neid, Argwohn, Zwietracht verrannt [die er nur ersetzen möchte durch] grosse, freudige Ambitionen, Freiheit, Wissen, Allumfassendes.» Wir wünschen ihm, dass er es schafft. 